\title{
Variables con posible capacidad modificadora del PSA ajustado al volumen de la zona transicional
}

\author{
J. Jara Rascón, D. Subirá Ríos, E. LLedó García, J.I. Martínez Salamanca, I. Moncada Iribarren, \\ R. Cabello Benavente, C. Hernández Fernández
}

Servicio de Urología. Hospital General Universitario Gregorio Marañón. Madrid.

Actas Urol Esp 2005; 29 (5): 473-480

\section{RESUMEN}

VARIABLES CON POSIBLE CAPACIDAD MODIFICADORA DEL PSA AJUSTADO AL VOLUMEN DE LA ZONA TRANSICIONAL

Objetivo: Determinar el efecto de la edad, los hallazgos del tacto rectal y el volumen prostático sobre el valor del PSA ajustado a la zona transicional (PSA-ZT) en la detección de cáncer prostático.

Material y métodos: Se analizan los datos recogidos en 243 pacientes biopsiados por sospecha de cáncer de próstata presentando un rango de PSA entre $4-20 \mathrm{ng} / \mathrm{ml}$ en los que se encontró presencia de cáncer en 62 casos (24.8\%). El volumen prostático total y el correspondiente a la zona transicional fueron calculados mediante ecografía transrectal aplicando la fórmula del elipsoide.

Resultados: Aplicando el análisis de regresión lineal no se encontró correlación entre la edad y el PSAZT (coeficiente de Pearson 0,00). Dividiendo a estos pacientes entre aquellos que tenían un tacto rectal normal (84\%) y los que presentaban hallazgos sugestivos de neoplasia (16\%), no se encontraron puntos de corte del PSA-ZT diferentes mediante el análisis de curvas ROC para obtener una sensibilidad del 95\%, variando la especificidad entre ambos grupos sólo entre un 24 a 26\% respectivamente. El tamaño prostático (menor o mayor de 40 cc) sí mostró que, para mantener una misma sensibilidad de 95\% en la detección de cáncer, el valor del PSAZT debería haber sido modificado, siendo de 0,17 en las próstatas $>40$ cc y de 0,25 en las de volumen $\leq 40$ cc.

Conclusiones: El valor del PSA-ZT, utilizado como parámetro potencialmente predictor de cáncer prostático, no pareció modificarse con la edad ni necesitó ser modificado en base a los datos aportados por el tacto rectal. Para mantener la sensibilidad del punto de corte óptimo de esta prueba, éste debería ser modificado en base al volumen prostático total.

Palabras clave: Cáncer prostático. Antígeno específico prostático. Volumen prostático.

\section{ABSTRACT}

PARAMETERS WITH POSSIBLE INFLUENCE IN PSA ADJUSTED FOR TRANSITION ZONE VOLUME

Objective: To evaluate the effect of age, digital rectal examination results and prostatic volume on PSA value adjusted to transition zone (PSA-TZ) in the detection of prostatic cancer.

Material and methods: Data of 243 patients with serum PSA of 4 to $20 \mathrm{ng} / \mathrm{ml}$ who underwent biopsy because of prostatic cancer suspicion are analyzed. In this population, cancer was detected in 62 cases (24.8\%). Total prostatic volume and transition zone volume were calculated by transrectal echography applying the ellipsoid formula.

RESULTS: Applying lineal regresion analysis, it was found no correlation between age and PSA-TZ (Pearson coefficient 0,00 ). By dividing these patients among those with normal rectal examination (84\%) and those with suspicious digital rectal examination (16\%), cutoff values of PSA-TZ were found to be not different by ROC curves analysis for $95 \%$ sensitivity varying specificity only among 24 and $26 \%$ between these two groups of patients. Prostatic size $\leqq$ or $>40 \mathrm{cc}$ ) showed that, for obtaining the same 95\% sensitivity in the detection of cancer, PSA-TZ value would require to be modified, being 0.17 in large prostates $(>40 \mathrm{cc}$ ) and 0.25 in small prostates $(\leq 40 \mathrm{cc})$.

Conclusions: The utility of PSA-TZ as a potential predictor parameter of prostatic cancer did not need to be modified with respect to age or to data of digital rectal examination. However, for supporting sensivity of its best cutoff value, PSA-TZ would need to be modified with respect to total prostatic volume. 
$\mathrm{I}$ ntentando mejorar la especificidad del PSA en la detección del cáncer prostático, diversos estudios encuentran que la determinación del PSA ajustando su valor a la zona transicional de la próstata (expresado en $\mathrm{ng} / \mathrm{ml} / \mathrm{cc}$ ) puede lograr este propósito ahorrando así un importante número de biopsias innecesarias. De hecho, la eficacia de este nuevo parámetro ha sido ratificada no sólo frente al PSA sino también frente al PSA densidad (PSAD) ${ }^{1,2}$ aunque no de modo unívoco en todos los trabajos. Así, algunos autores no encuentran una clara superioridad del PSA$Z \mathrm{~T}$ frente al $\mathrm{PSAD}^{3,4}$ mientras que otros refieren que su especificidad, en pacientes con PSA en el rango de 4 a $10 \mathrm{ng} / \mathrm{ml}$, es mejor también que la encontrada con el PSA libre/total ${ }^{5}$.

Asimismo, se han realizado estudios investigando la utilidad de este nuevo parámetro sobre la indicación de realizar biopsias sucesivas después de un primer resultado negativo, como el coordinado por Horinaga sobre 144 pacientes $^{6}$, encontrando que, para una misma sensibilidad del 90\%, el PSA-ZT fue capaz de alcanzar una especificidad del 46\%, mientras que el PSAD, el PSA libre/total y el PSA total sólo lograron una especificidad del 36\%, 18\% y $25 \%$ respectivamente.

Sin embargo, a pesar de que se ha postulado que el PSA-ZT puede ser la variable PSA-relacionada capaz de predecir la presencia de cáncer con mejor idoneidad diagnóstica ${ }^{5}$, aún no existen datos concluyentes respecto a cual debe ser el punto de corte óptimo capaz de alcanzar una sensibilidad del 90-95\% en la mayor parte de los sujetos sometidos a biopsia con un PSA entre 4$20 \mathrm{ng} / \mathrm{ml}$, lo que correspondería a la mayor parte de los pacientes biopsiados actualmente. Debido a esto, el presente estudio intenta valorar si variables tales como la edad de los pacientes, un tacto rectal sospechoso de neoplasia o el volumen prostático pueden condicionar la modificación de los valores de PSA-ZT capaces de alcanzar su máxima eficacia diagnóstica.

\section{MATERIAL Y MÉTODOS}

Se incluyeron en el presente estudio todos aquellos pacientes con sospecha de cáncer prostático a los que se realizaron biopsias prostáticas sextantes ecodirigidas que presentaban valores de PSA en el rango de 4-20 ng/ml descartándose todos aquellos que presentaban antecedentes capaces de modificar esta determinación analítica. El tacto rectal, realizado en todos los casos, se categorizó como normal o sospechoso de cáncer por el mismo urólogo que realizó la ecografía transrectal.

Se determinó el valor de PSA total en el suero de los pacientes en todos los casos antes de la realización de la biopsia analizando la muestra obtenida mediante un método inmunométrico equimolar de quimioluminiscencia (Immulite 2000 PSA, Diagnostic Products Corporation, Los Ángeles, USA). La fracción PSA libre/total fue analizada de modo similar mediante el correspondiente ensayo secuencial inmunométrico (Immulite 200 free PSA). La ecografía transrectal de la próstata fue realizada usando un ecógrafo tipo Diagnostic Ultrasound System Brüel \& Kjaer modelo 3535 con un transductor transrectal multiplanar de $7 \mathrm{MHz}$ con canal de biopsia integrado tipo 8551. Antes de la realización de esta prueba diagnóstica se pautó profilaxis antibiótica con ciprofloxacino $500 \mathrm{mg}$ V.O. en dos tomas, la primera 2 horas antes de la punción y la segunda 12 horas después. Se evaluó la imagen obtenida de la próstata en los planos transverso y sagital con el paciente en posición de decúbito lateral.

El volumen prostático fue determinado mediante el software integrado del ecógrafo aplicando la fórmula del elipsoide, dividiéndose los pacientes estudiados entre los que presentaban una próstata menor de 40 cc y aquellos con próstatas mayores de $40 \mathrm{cc}$. La anchura (diámetro transversal) y la altura (diámetro anteroposterior) fueron medias en el plano transversal en la posición correspondiente a sus mayores dimensiones. La longitud (diámetro cefalocaudal) fue determinada en el plano sagital midiendo el eje de la línea media desde la base hasta el ápex prostático. El volumen de la zona transicional fue medido también utilizando la misma fórmula del elipsoide y en los mismos planos que el volumen prostático total. El PSAD, expresado en $\mathrm{ng} / \mathrm{ml} / \mathrm{cc}$, fue calculado dividiendo el PSA total entre el volumen prostático hallado. El PSA-ZT fue definido como el PSA dividido por el volumen de la zona transicional y expresado en las mismas unidades que el PSAD. 
En todos los pacientes las biopsias prostáticas ecodirigidas fueron obtenidas mediante el método propuesto por Hodge ${ }^{7}$ con realización de biopsias sextantes randomizadas en la zona periférica de la próstata utilizando para ello una aguja de punción automática y una aguja de calibre 18-gauge a través del canal de biopsia. El número de biopsias randomizadas realizadas fue de seis en cada caso independientemente del volumen prostático presente, con el fin de poder evaluar de modo uniforme los resultados obtenidos.

Se realizó el análisis estadístico de los datos encontrados mediante test de la $\mathrm{t}$ de Student según los tipos de variables comparadas. Los coeficientes de correlación para la comparación de variables cuantitativas se calcularon mediante análisis de regresión lineal. Se determinaron las curvas ROC (receiver operating characteristic) valorando la sensibilidad frente a especificidad para diferentes puntos de corte de la variable PSA-ZT, calculándose el área bajo la curva obtenida en cada caso y realizándose la comparación entre las áreas según el test de Hanley-McNeil ${ }^{8}$. El nivel de significación estadística se estableció en 0,5. Para el adecuado manejo estadístico de estos datos se utilizó el programa informático SPSS en su versión 10 .

\section{RESULTADOS}

Un total de 249 pacientes se consideraron válidos para su análisis. La media de edad fue $67,7 \pm 6,7$ años (desviación típica) con un rango de 49-84 años. Del total de pacientes, el tacto rectal fue considerado sospechoso de malignidad en 40 casos $(16,1 \%)$ y de carácter no sospechoso en 209 (83,9\%). Se detectó cáncer prostático en 62 casos $(24,9 \%)$ correspondiendo los 187 casos restantes $(75,1 \%)$ a diagnóstico histopatológico de benignidad.

La tasa de detección de cáncer fue valorada, primeramente, segmentando la muestra total de pacientes estudiados entre aquellos que presentaron un tacto rectal sugestivo de benignidad y aquellos cuya exploración rectal sí presentaba caracteres sospechosos de malignidad. Se analizaron las curvas ROC del PSA-ZT en los pacientes con tacto indicativo de benignidad y en los casos con tacto sospechoso de neoplasia. Las áreas bajo la curva obtenidas, en los pacientes con tacto rectal normal o anormal, fueron respectivamente 0,727 y 0,674 con unos valores de intervalo de confianza al $95 \%$ superponibles entre ambas curvas. El punto de corte indicado para obtener una sensibilidad del $95 \%$ en ambos grupos fue similarmente $0,18 \mathrm{ng} / \mathrm{ml} / \mathrm{cc}$ con una especificidad respectiva del $26 \%$ y del $24 \%$ pudiéndose deducir que la información aportada por el tacto rectal no tuvo impacto significativo en los resultados obtenidos sobre la detección de cáncer mediante el PSA-ZT (Fig.1).

En segundo lugar, puesto que tanto la edad como el volumen de la zona transicional prostática son variables cuantitativas, se llevó a cabo un análisis de regresión lineal en esta serie, entre las determinaciones de PSA-ZT encontradas en los pacientes sin cáncer y la edad presentada por

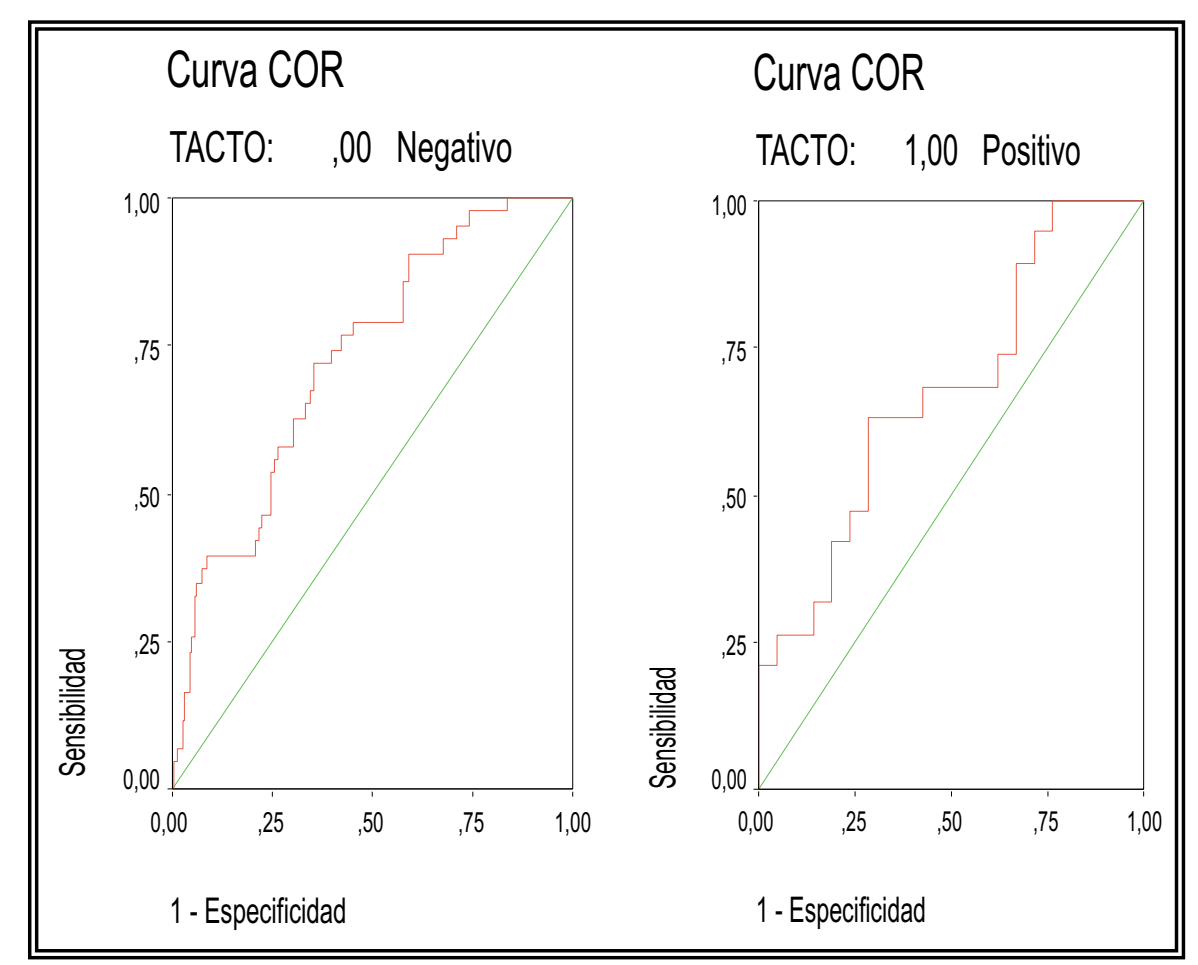

FIGURA 1. Curvas de rendimiento diagnóstico (ROC) del PSA-ZT para la detección de cáncer. A. En casos con tacto rectal negativo. B. En casos con tacto rectal sospechoso. 
estos pacientes. Se intentó así estudiar si el factor edad por sí solo se podía correlacionar con la determinación del PSA-ZT como posible variable modificadora. Sobre un total de 187 casos sin cáncer, tal como se muestra en la Figura 2 se observó que el aumento de la edad no mostró ninguna correlación con las cifras de PSA-ZT (coeficiente de Pearson 0,00) no aportando, por tanto, ninguna información la variable edad sobre posibles modificaciones en el PSA-ZT.

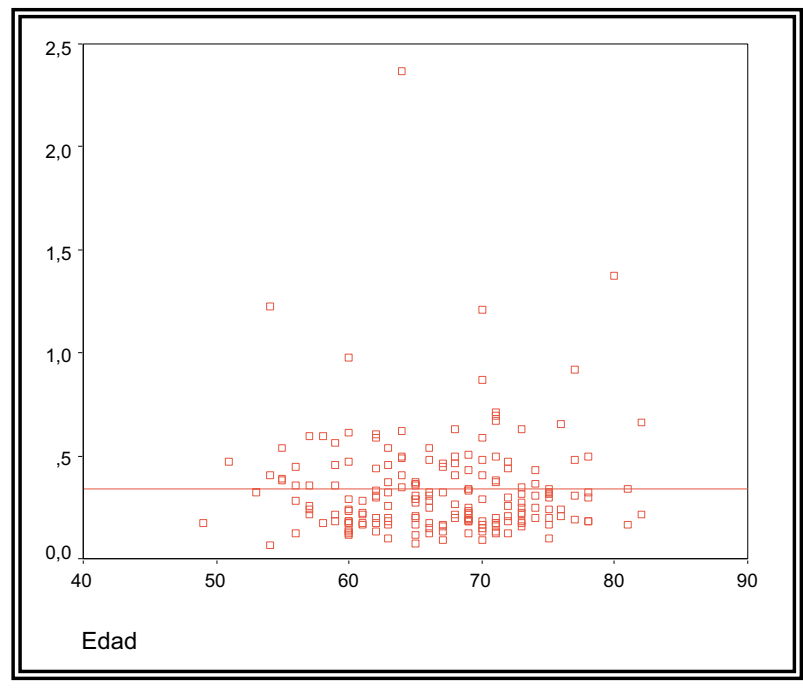

FIGURA 2. Representación gráfica la ausencia de correlación encontrada entre los niveles de PSA-ZT y el aumento de la edad en los pacientes sin cáncer (187 casos).

En tercer lugar, con el fin de analizar si la detección de cáncer mediante un mismo número de biopsias en todos los casos podría verse dificultada en aquellos pacientes de mayor volumen prostático, se compararon estadísticamente mediante la t de Student las medias del volumen prostático total y del volumen de la zona transicional obtenidas entre los casos con diagnóstico histológico de benignidad y los diagnosticados de cáncer en dichas biopsias. Se encontró que la media de ambos volúmenes fue significativamente menor $(\mathrm{p}<0,0001)$ en los casos de cáncer respecto a los casos diagnosticados como patología benigna, concluyéndose que el menor tamaño de la próstata puede haber facilitado el diagnóstico en estos casos (Tabla 1).

Posteriormente, para determinar en base a los datos recogidos si el punto de corte del PSAZT capaz de lograr una sensibilidad del 95\%,
Tabla 1

Estadística descriptiva del volumen prostático total y del volumen transicional en el grupo total de pacientes

\begin{tabular}{lccc}
\hline & $\begin{array}{c}\text { Patología } \\
\text { Benigna }\end{array}$ & Cáncer & Valor p* \\
\hline $\begin{array}{l}\text { Volumen } \\
\text { total (cc) }\end{array}$ & & & \\
Media & 53,82 & 39,92 & $<0,0001$ \\
Mediana & 51,30 & & 36,25 \\
Desv tip. & 21,32 & 18,07 & \\
$\quad$ IC 95\% & $51,46-56,17$ & $36,56-43,27$ & \\
& & & \\
Volumen & & & \\
transicional (cc) & & & \\
$\quad$ Media & 32,54 & 22,50 & $<0,0001$ \\
$\quad$ Mediana & 29,80 & 20,20 & \\
$\quad$ Desv tip. & 14,89 & 12,84 & \\
$\quad$ IC 95\% & $29,86-35,23$ & $18,08-26,91$ & \\
\hline
\end{tabular}

* $t$ de Student comparando valores de medias entre casos con y sin cáncer

IC 95\% = Intervalo de confianza del 95\% de las medias.

estimado en $0,18 \mathrm{ng} / \mathrm{ml} / \mathrm{cc}$, debería ser modificado o no según el tamaño de la próstata, se fragmentó la serie total de pacientes en dos grupos, adoptando el término de "próstatas pequeñas" para las próstatas de volumen $>40 \mathrm{cc}$ y aceptando como "próstatas grandes" las de volumen $>40 \mathrm{cc}$. Entre las próstatas pequeñas en $31 / 79$ casos (39,2 \%) se obtuvo el diagnóstico de cáncer mientras que en las mayores de $40 \mathrm{cc}$ sólo se llegó a este diagnóstico en 31/170 casos $(18,2 \%)$. Se realizaron curvas ROC para cada uno de estos subgrupos (Fig. 3) determinando el área bajo la curva para el PSA-ZT en relación al diagnóstico de cáncer, intentando ver si existían diferencias en el punto de corte del 95\% entre los dos grupos de pacientes con próstatas de diferente tamaño. Los resultados obtenidos, con un área bajo la curva de 0,714 para las próstatas de volumen $<40$ cc y de 0,672 para las de volumen $\geq 40 \mathrm{cc}$ ( $<<0,001)$, muestran que para mantener la misma sensibilidad del 95\% el punto de corte óptimo del PSA-ZT debería ser modificado según el volumen presentado, pasando a ser 0,17 en las próstatas $\geq 40$ cc y 0,25 en las de volumen $\leq 40 \mathrm{cc}$. Se repitió el mismo análisis en el subgrupo de pacientes con PSA entre 4 y $10 \mathrm{ng} / \mathrm{ml}$ encontrándose idénticos puntos de corte para un $95 \%$ de sensibilidad variando la especificidad obtenida (Tabla 2). 


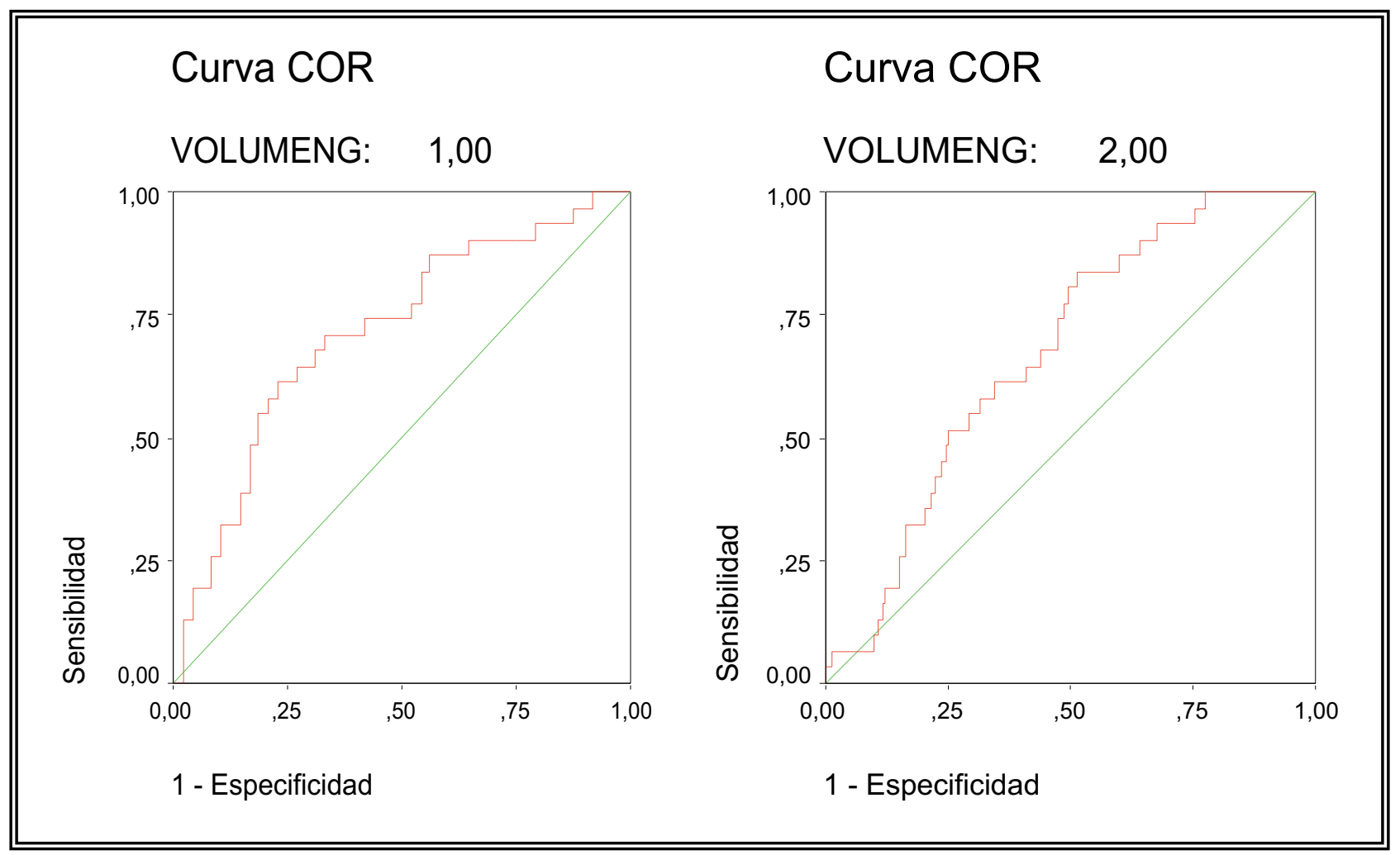

FIGURA 3. Curvas ROC obtenidas para el PSA-ZT respecto a la detección de cáncer según el volumen prostático presentado. A. Casos con volumen < $40 \mathrm{cc}$. B. Casos con volumen $>40 \mathrm{cc}$.

Tabla 2

Variación de puntos de corte del PSA-ZT según el volumen prostático total

\begin{tabular}{lccc}
\hline $\begin{array}{l}\text { Volumen } \\
\text { Prostático }\end{array}$ & $\begin{array}{c}\text { No } \\
\text { pacientes } \\
\mathbf{9 5 \%}\end{array}$ & $\begin{array}{c}\text { Punto de } \\
\text { corte } \\
\text { Sensibilidad }\end{array}$ & $\begin{array}{c}\% \\
\text { correspondiente } \\
\text { Especificidad }\end{array}$ \\
\hline $\begin{array}{l}\text { En el grupo } \\
\text { totalå }\end{array}$ & & & \\
$<40 \mathrm{cc}$ & 79 & $0,25 \mathrm{ng} / \mathrm{ml} / \mathrm{cc}$ & $13 \%$ \\
$>40 \mathrm{cc}$ & 170 & $0,17 \mathrm{ng} / \mathrm{ml} / \mathrm{cc}$ & $23 \%$ \\
& & & \\
En el grupo con & & & \\
PSA 4-10 ng/ ml & & & \\
$<40 \mathrm{cc}$ & 53 & $0,25 \mathrm{ng} / \mathrm{ml} / \mathrm{cc}$ & $18 \%$ \\
$>40 \mathrm{cc}$ & 103 & $0,17 \mathrm{ng} / \mathrm{ml} / \mathrm{cc}$ & $35 \%$ \\
\hline
\end{tabular}

\section{DISCUSION}

Aunque se acepta ampliamente que el PSA es el marcador tumoral más importante en la detección de cáncer prostático, la falta de especificidad de esta determinación analítica, como ya se ha apuntado previamente, condiciona un número de biopsias negativas para cáncer que, utilizando el punto de corte usualmente acepta- do de $4 \mathrm{ng} / \mathrm{ml}$, se sitúa alrededor de las dos terceras partes de las biopsias realizadas ${ }^{9}$. Por ello se siguen actualmente realizando estudios intentando mejorar su adecuación diagnóstica especialmente en el rango de PSA entre 4 y 10 $\mathrm{ng} / \mathrm{ml}$, en el que es mayor el solapamiento entre el diagnóstico de cáncer y el de HPB. La introducción del PSA-ZT, objeto de este estudio, responde a este intento de mejora en el rendimiento diagnóstico del PSA sérico total. Sin embargo, las divergencias observadas entre diferentes trabajos al definir cual debe ser el punto de corte óptimo de esta última prueba, oscilando desde 0,35 hasta $0,15 \mathrm{ng} / \mathrm{ml} / \mathrm{cc}^{10-12}$ hacen pensar que, quizás, existan diferencias en las características poblacionales de las muestras estudiadas que puedan condicionar estos diferentes resultados. Entre estas características podrían encontrarse la diferente edad de los pacientes incluidos, diferencias entre los hallazgos del tacto rectal y diferentes volúmenes prostáticos medios de las muestras estudiadas. 
En nuestro estudio, segmentando los pacientes en base a la presencia o no de un tacto rectal sospechoso de malignidad, no se encontró diferencia al valorar las curvas ROC del PSA-ZT para la detección de cáncer. El punto de corte del PSAZT en ambos grupos para lograr una sensibilidad del 95\% coincidió en $0,18 \mathrm{ng} / \mathrm{ml} / \mathrm{cc}$ mostrando una especificidad similar entre ambos grupos ( $26 \%$ y $24 \%$ respectivamente). Aunque se encontraron diferencias estadísticamente significativas entre las medias de PSA-ZT entre ambos grupos de pacientes, los intervalos de confianza del 95\% obtenidos fueron superponibles por lo que el tacto rectal no pareció ser una variable modificadora del valor del PSA-ZT en su capacidad predictora de cáncer de próstata.

Estos datos coinciden con los obtenidos por Djavan y cols. ${ }^{13}$, quienes encuentran que el PSAZT se muestra como una variable independiente de los hallazgos del tacto rectal, no encontrando tampoco ningún impacto valorable estadísticamente de dicha exploración rectal sobre el PSA, el PSAD o el PSA libre/total en su capacidad de detectar el cáncer de próstata. De hecho, se admite comúnmente que las elevaciones de PSA por encima de un punto de corte considerado como válido, usualmente situado en el valor de 4 $\mathrm{ng} / \mathrm{ml}$, requieren la realización de biopsia independientemente de si el tacto rectal encuentra o no signos indicativos de presencia de cáncer.

Respecto a la posible influencia de la edad de los pacientes en los resultados de los diversos trabajos publicados, parece interesante mencionar que en el estudio multicéntrico coordinado por Djavan $^{14}$, sobre 974 pacientes incluidos con PSA entre 4 y $10 \mathrm{ng} / \mathrm{ml}$, se encontró que el PSA total, el PSA-ZT y el PSAD fueron significativamente más altos en los pacientes con cáncer prostático que en aquellos sin cáncer en los correspondientes grupos de edad. En los pacientes con cáncer, el PSA libre/total fue significativamente más bajo $(\mathrm{p}<0,001)$ dentro de los mismos rangos de edad. Todos estos parámetros estudiados no se afectaron por la estratificación según edades encontrándose que el PSA-ZT y el PSA libre/total aparecían como los mejores parámetros predictores de cáncer. El cociente PSA libre/total no pareció guardar relación con el volumen prostático, el volumen de la ZT ni con la edad.
En el presente estudio, la edad tampoco apareció como una variable capaz de correlacionarse con los niveles de PSA-ZT en el análisis de regresión lineal simple realizado sobre 187 casos sin cáncer en la que la medición de esta variable pudo ser realizada (coeficiente de Pearson 0,00). Esto puede explicarse por el aumento del tamaño de la próstata que suele observarse de modo paralelo al incremento de la edad de modo que, si bien es esperable un aumento del PSA total con la edad, también se puede esperar un aumento simultáneo del volumen de la zona transicional, por lo que el cociente entre ambos parámetros, que es lo que define al PSA-ZT, finalmente es esperable que no resulte afectado.

Finalmente, el otro factor que también podría influenciar el valor del PSA-ZT es el volumen prostático. Éste, como es bien sabido, es un importante factor causal de la elevación de los niveles de PSA en ausencia de adenocarcinoma, condicionando la decisión de biopsiar y, por supuesto, de rebiopsiar a los pacientes cuando ya tenemos una biopsia inicial negativa. Dado que la muestra obtenida en las biopsias sextantes solamente aporta $90 \mathrm{~mm}$ de tejido prostático $(6 \mathrm{x}$ $15 \mathrm{~mm}$ de cada muestra) para su estudio, cuanto mayor sea un volumen prostático menor será la probabilidad de detectar un cáncer de pequeño tamaño. Debido a esto, mayoritariamente se acepta el papel del volumen prostático total como un factor a tener en cuenta al interpretar el resultado de las biopsias, pero la importancia de la ZT ha sido mucho menos frecuentemente investigada. Sin embargo, un estudio determinando la correlación entre PSA y volumen de la ZT mostró que el mejor predictor del nivel de PSA sérico no es el volumen prostático total sino el volumen de la ZT, especialmente el volumen de la zona epitelial transicional ${ }^{15}$. Por otra parte, la ecografía transrectal muestra diferencias objetivables en la proporción del volumen transicional respecto al volumen prostático total en los hombres con y sin HPB, implicando que deben existir también diferencias entre PSA-ZT y PSAD ${ }^{16}$.

Además, existen estudios como el llevado a cabo por Moon en $2000^{17}$ en el que, sobre 105 hombres estudiados, usando un análisis de regresión lineal se encontró una buena correlación entre el volumen de la ZT y el volumen total 
de la glándula prostática tanto en los pacientes con próstatas grandes (> $40 \mathrm{cc}$ ) como en los de próstatas de menor tamaño ( $<40 \mathrm{cc}$ ). En el subgrupo de pacientes con próstatas pequeñas, usando un umbral de PSA-ZT de 0,35 se pudo haber reducido el número de biopsias en un $34 \%$ detectándose un $76 \%$ de cánceres. En los casos con próstatas mayores, un umbral de PSA-ZT de 0,30 habría reducido el número de biopsias a un $43 \%$ detectando así un $82 \%$ de cáncer. Estos mismos autores también analizaron simultáneamente el papel del PSA libre/total en relación al volumen de la glándula prostática notificando que esta determinación fue hallada más efectiva en las próstatas más pequeñas que en las de mayor tamaño con un punto de corte de $12 \%$ en las próstatas menores de $40 \mathrm{cc}$ y de $10 \%$ en las mayores de $40 \mathrm{cc}$.

A partir de los datos del presente estudio, también encontramos que los pacientes con cáncer de la muestra estudiada presentaron un valor medio de volumen prostático total inferior a los casos diagnosticados como patología benigna $(\mathrm{p}<0,0001)$ y esta diferencia se mantuvo igualmente al medir el volumen de la ZT entre ambos grupos siendo el valor medio de la ZT $22,5 \mathrm{cc}$ en los casos de cáncer y 32,5 cc en los correspondientes a patología benigna. Por otra parte, los puntos de corte del PSA-ZT para lograr una misma sensibilidad del 95\% también variaron según el volumen prostático, situándose en 0,25 $\mathrm{ng} / \mathrm{ml} / \mathrm{cc}$ en el subgrupo con próstatas de volumen inferior a $40 \mathrm{cc}$ y disminuyendo a 0,17 $\mathrm{ng} / \mathrm{ml} / \mathrm{cc}$ en los casos con próstatas mayores de 40 cc. Asimismo, estos puntos de corte se mantuvieron sin cambios en los pacientes con PSA en el rango de 4-10 $\mathrm{ng} / \mathrm{ml}$. Utilizando como punto de corte para el volumen prostático el valor de 30 cc, Djavan y cols. ${ }^{13}$ informaron, como conclusión de un estudio sobre 559 casos con PSA de 4-10 $\mathrm{ng} / \mathrm{ml}$, que el cociente PSA libre/total se demostró como un indicador más efectivo en próstatas de $<30$ cc y que el PSA-ZT fue encontrado más efectivo en próstatas de $>30$ cc proponiéndose la utilización de estas dos variables conjuntamente para aumentar las posibilidades de predecir adecuadamente la existencia del cáncer de próstata.

Adicionalmente, el estudio de Taneja y cols. ${ }^{2}$ también menciona que, teniendo en cuenta el volumen prostático, la mayor discrepancia entre el PSA-ZT y el PSAD se observa en las próstatas de menos de $30 \mathrm{cc}$, ya que manteniendo una sensibilidad del 95\% para ambas variables, la especificidad obtenida a un punto de corte óptimo en cada caso varió de un $28,2 \%$ para el PSA-ZT a un 5,6\% para el PSAD. Estos autores concluyen que, incluso aunque se estandaricen correctamente diferentes puntos de corte del PSA-ZT estratificando a los pacientes según su volumen prostático, la aplicación de estos parámetros estará siempre sujeta a la variabilidad de las medidas del volumen de la próstata y de la ZT. Se necesitaría, por tanto, un método estandarizado para definir estas medidas volumétricas favoreciendo así la reproducibilidad de los hallazgos obtenidos por diferentes estudios.

Al valorar la utilidad del PSA-ZT en nuestro estudio, se debe tener en cuenta que los resultados obtenidos muestran que para mantener la sensibilidad del 95\% al evaluar próstatas de diferente tamaño el punto de corte del PSA-ZT debería ser modificado según el volumen presentado. Este hallazgo implica que el PSA-ZT puede no ser un test eficiente en las poblaciones de screening en los que muchas de las próstatas estudiadas son de tamaño normal o se encuentran levemente aumentadas. Ciertamente, se debería distinguir entre pacientes reclutados en programas de screening y aquellos referidos al especialista por presentar sintomas urinarios del tracto urinario inferior o con sospecha de padecer un cáncer prostático. De modo similar, cuando se analizan estudios contradictorios sobre el valor del PSAD, parece que el mayor beneficio se demuestra usualmente en el grupo de pacientes referidos por síntomas sugestivos de prostatismo o con sospecha de cáncer en el tacto rectal realizado ${ }^{18}$. De acuerdo a nuestros datos el uso del PSA-ZT parece más eficiente en hombres con próstatas mayores de 40 cc, donde muestra una especificidad aumentada.

Como conclusión, se puede afirmar que el PSA-ZT, en la muestra estudiada, no pareció ser un valor dependiente de los hallazgos del tacto rectal ni de la edad. En cambio, sí debió modificarse su punto de corte óptimo en función del volumen prostático (menor o mayor de $40 \mathrm{cc}$ ), lo que debería ser tenido en cuenta al plantearse su posible aplicación clínica. 


\section{Agradecimientos}

A la Dra. Dolores Vigil Escribano y a José María Bellón Cano del Servicio de Medicina Preventiva y Gestión de Calidad por su inestimable y desinteresada ayuda en el procesamiento estadístico de los datos de este estudio.

\section{REFERENCIAS}

1. Kurita Y, Ushiyama T, Suzuki K, Fujita K, Watanabe K. PSA value adjusted for the transition zone volume in the diagnosis of prostate cancer. Int $J$ Urol 1996;3:367-372.

2. Taneja S, Tran K, Lepor H. Volume specific cutoffs are necessary for reproducible application of prostate specific antigen density of the transition zone in prostate cancer detection. Urology 2001;58: 222-227.

3. Aksoy Y, Oral A, Aksoy H, Demirel A, Akcay F. PSA density and PSA transition zone density in the diagnosis of prostate cancer PSA gray zones. Ann Clin Lab Sci 2003;33: 320-323.

4. Rietbergen JBW, Kranse R, Hoedemaeker RF, et al. Comparison of prostate specific antigen corrected for total prostate volume and transition zone volume in a population based screening study. Urology 1998;52:237-246.

5. Kikuchi E, Nakashima J, Ishibashi M. Prostate specific antigen adjusted for transition zone volume. The most powerful method for detecting prostate carcinoma. Cancer 2000;89: 842-849.

6. Horinaga M, Nakashima $\mathrm{J}$, Ishibashi $\mathrm{M}$, et al. Clinical value of prostate specific antigen based parameters for the detection of prostate cancer on repeat biopsy: the usefulness of complexed prostate specific antigen adjusted for transition zone volume. J Urol 2002;168:986-990.

7. Hodge KK, Mcneal JE, Terris MK, et al. Random systematic versus directed ultrasound guided transrectal core biopsies of the prostate. J Urol 1989;142:71-75.

8. HANLEY JA, MCNEIL BJ. A method of comparing the areas under receiver operating characteristic curves derived from the same cases. Radiology 1983;148:839-843.

9. Brawer MK, Beattie J, Wener MH, Vessella RL, Preston SD, Lange PH. Screening for prostatic carcinoma with PSA: Results of the second year. J Urol 1993;150:106-112.

10. Zlotta AR, Djavan B, Marberger M, Schuman CC. Prostate specific antigen density of the transition zone: a new effec- tive parameter for prostate cancer detection. J Urol 1997; 157:1315-1321.

11. Djavan B, Remzi M, Zlotta AR, et al. Complexed prostate specific antigen, complexed prostate specific antigen density of total and transition zone, complexeed/total prostate specific antigen ratio, free-to-total prostate specific antigen ratio, density of total and transition zone prostate specific antigen: results of the prospective multicenter European trial. Urology 2002;60(Suppl 4A):4-9.

12. Allepuz C, Sanz JI, Carela J, Benejam R, García R, Rioja LA Aportación del PSAD y PSA-AD en el diagnóstico precoz del cáncer de próstata. Actas Urol Esp 1997;21:843-851.

13. Djavan B, Zlotta AR, Byttebier G, et al. Prostate specific antigen density of the transition zone for early detection of prostate cancer. J Urol 1998;160:411-419.

14. Djavan B, Zlotta AR, Remzi M, et al. Total and transition zone prostate volume and age: How do they affect the utility of PSA-based diagnostic parameters for early prostate cancer detection?. Urology 1999;54:846-852.

15. Lepor H, Wang B, Shapiro E: Relationship between prostatic epithelial volume and serum prostate specific antigen levels. Urology 1994;44:199-205.

16. Greene DR, Egawa S, Hellerstein DK, Scardino PT. Sonographics measurements of the transition zone of the prostate in men with and without benign prostatic hyperplasia. Urology 1990;36:293-299.

17. Moon DG, Lee JG, Kim JJ, Koh SK, Cheon J The influence of prostate volume on the prostate specific antigen (PSA) level adjusted for the transition zone volume and free-tototal PSA ratio: a prospective study. BJU International 2000;86:670-674.

18. Bazinet M, Meshref AW, Trudel C, et al. Prospective evaluation of prostate specific antigen density and systematic biopsies for early detection of prostatic carcinoma. Urology 1994;43:44-49.

Dr. J. Jara Rascón

C/ Condado de Treviño no 2 , p-1, 5ํ- C 28033 Madrid

(Trabajo recibido el 26 octubre de 2004) 